\title{
Daño al futuro: ¿puede el no comparativismo resolver el problema de la no-identidad?
}

\author{
Harm to future: Could the No Comparativism solve the Non- \\ Identity Problem?
}

SANTIAGO TRUCCONE BORGOGNO*

\begin{abstract}
Resumen: la tesis clásica del daño afirma que un sujeto daña a otro cuando lo coloca en una peor situación de la que podría estar de otro modo. Sin embargo, algunas acciones causan consecuencias malas en determinados sujetos pero no los colocan en una condición peor de la que podrían estar de otro modo. En tales casos el no-comparativismo parece poder aportar la solución correcta desde que, para tales tesis dañar a otro es colocar a un sujeto en una condición mala. En este trabajo se intentará mostrar que incluso tales aproximaciones son infructuosas, proponiendo, en su lugar una tesis combinada.

Palabras clave: daño - problema de la no-identidad - no-comparativismo
\end{abstract}

\begin{abstract}
: classic harm accounts assert that someone harms other person if the agent cases the victim to be worse off than he would have been. However, some behaviour causes some bad outcomes in certain persons, but does not make that person worse off. In these cases the non-comparativism seems to provide the correct answer. On that thesis someone harms another person if the agent causes that the victim to be in a bad state. In this work, I will try to show that even that kind of theories can't solve the non-identity problem. Instead, I try to develop a combined theory.

Keywords: harm - non identity problem no-comparativism.
\end{abstract}

\section{Introducción}

Ciertas conductas pueden causar que algunas personas sean agraviadas o estén en una condición dañada en el futuro. Estos pueden ser malos efectos que parecen darnos razones morales para no realizar tales conductas. Sin embargo, en algunos casos es predecible que, si nosotros no hubiéramos realizado las conductas que producen consecuencias disvaliosas en un ser, esas personas particulares nunca existirían. Por lo tanto conocemos que, si realiza-

Fecha de recepción: 17/04/2015. Fecha de aceptación: 19/07/2015.

* Miembro del Programa en Ética y Teoría Política (FDyCS-UNC [https://http://www.programadeetica.com.ar]); Becario Doctoral CONICET-Argentina; Magister en Derecho Penal (U. Salamanca); Abogado (U. Nacional de Córdoba-Argentina); Líneas de Investigación: Ética y Generaciones Futuras - Justificación de Prohibiciones Penales. "Derecho Penal y Obligaciones hacia las generaciones futuras: Primeras líneas de Justificación" XIV Anuario del Centro de Investigaciones Jurídicas y Sociales- FDyCS-UNC, La Ley 2014, pp.205-228; "Delitos Acumulativos Ambientales: una aproximación desde el Republicanismo" Revista de Derecho Ambiental de la Universidad de Palermo, 2013, pp.59-98. Santiagotruccone@gmail.com 
mos tales acciones, ello no será peor para esas personas futuras. ¿Elimina este hecho nuestra razón moral para no realizar tal conducta? (Parfit, 1986, 854). Creemos que la respuesta a tal interrogante es negativa. Sin embargo, es sumamente dificultoso poder articular una respuesta coherente que dé cuenta de qué es lo malo en la realización de tales acciones. Esta situación se conoce en la literatura como problema de la no-identidad. Problema así denominado por Derek Parfit ${ }^{1}$ dado que de acuerdo a cuál sea la acción que se realice, la identidad de la persona resultante será diferente. Imagínese el siguiente caso:

(Moni y Paola): una mujer, Moni, decide concebir a un niño. Ella concurre al médico para realizarse un chequeo. El médico le dice que tiene dos noticias para contarle, una buena y una mala. La mala noticia es que si Moni concibe ahora su niño tendrá una discapacidad. Tal discapacidad tendrá un impacto negativo en la calidad de vida del niño, aunque sin embargo no es tan grave para hacer de la vida del niño una tan mala que no valga la pena ser vivida. Supóngase, por ejemplo, que el niño nacerá sordo. La buena noticia es que Moni puede evitar tal discapacidad tomando una píldora todos los días durante los próximos dos meses. Si ella lo hace, y concibe dentro de dos meses, su niño será perfectamente saludable. Moni piensa que tomar la píldora por dos meses es ligeramente inconveniente para ella, por lo que decide concebir ahora. Como resultado nace Paola quien es sorda. ${ }^{2}$

Se han realizado varios intentos en orden a explicar por qué acciones de las del tipo que realiza Moni dañan a Paola. Las líneas que se han seguido para cumplir con tal tarea han sido principalmente dos: una comparativa y otra no comparativa. La primera, consiste en entender al daño en términos comparativos donde dañar, en la posición más comúnmente defendida, sería colocar a otro sujeto en una condición peor que aquella en la que podría haber estado de otro modo (Feinberg, 1994, 6). Esta aproximación no puede dar una explicación satisfactoria, dado que por ejemplo en nuestro caso, Paola no podría estar en una mejor condición si Moni no hubiese actuado como lo hizo. Si Moni hubiese esperado dos meses antes de concebir, el resultado sería que Paola no hubiese existido. ${ }^{3}$

1 El problema ha sido descubierto casi simultáneamente por Derek Parfit, Robert Adams y Thomas Schwartz. Derek Parfit ha sido quien ha denominado de este modo al problema en un manuscrito que hizo circular en 1976 "Overpopulatión: Part One". El argumento presentado apareció luego en dos artículos publicados en 1981 y 1982: "Energy and the Further Future" Center for Philosophy and Public Policy, University of Maryland (1981), pp.112-121 y "Future Generations: Further Problems" en Philosophy and Public Affair, Vol. 11, ํ 2 (Spring, 1982), 113-172. Alcanzó un desarrollo mayor, luego de que fuera planteado en 1984 con detalle en la parte IV de su libro Reasons and Persons, Oxford Clarendon Press, pp. 351 y Ss.

2 He modificado el ejemplo de Boonin (2014:2). El caso es muy similar a uno real ocurrido en 2002, en el cual Gauvin nació sordo por decisión de sus madres. Sharon Duchesneau y Candace McCullough decidieron concebir deliberadamente, realizando una serie de gestiones, a un hijo sordo, cuando tenían la posibilidad de concebir a uno sin esa afección. (http://elpais.com/diario/2002/04/09/sociedad/1018303205_850215.html)

3 Esto es así porque, independientemente de cuál sea la teoría de la identidad personal que se sostenga, lo cierto es que cada persona surge de un óvulo y un espermatozoide concreto. Si uno hubiese sido concebido unos segundos después de lo que lo hizo, el óvulo hubiese sido fecundado por un espermatozoide diferente, y ese niño hubiese compartido con el primero algunos de sus genes, pero no todos. Dado que en este caso el óvulo fecundado sigue siendo el mismo, todavía podría decirse que el sujeto es, más o menos, la misma persona. Esto porque se asume que hay casos donde las preguntas sobre la identidad no tienen que tener 
La misma conclusión insatisfactoria es aportada por otras posiciones comparativas que sólo cambian la línea de base en función de la cual se realiza la comparación. Así según la tesis comparativa temporal, un sujeto daña a otro cuando lo coloca en una condición que es peor que aquella en la que estaba antes (Raz, 1986, 414). Esta tesis también da resultados insatisfactorios en esta clase de casos dado que, Paola no está en ninguna condición peor que antes. Sencillamente, ella nace en esa condición. Parece que las propuestas comparativas no son la mejor opción a la hora de explicar por qué nuestras acciones pueden dañar a las generaciones futuras en casos atravesados por el problema de la no-identidad. Por tal razón, quizás sea más prometedor tomar la segunda vía de solución y proponer una tesis no comparativa del daño. En este trabajo se analizarán las dos defensas más robustas de tal clase de tesis. Se intentarán mostrar sus ventajas y desventajas. Finalmente en atención a las debilidades presentadas, se procurará defender una respuesta superadora.

\section{Tesis del daño no comparativas}

En líneas generales, las tesis no comparativas del daño niegan que las comparaciones sean relevantes para saber si se ha dañado a alguien o no. Ellas consideran que se ha dañado cuando se causa que una persona esté en mal estado (o tenga una condición mala). La idea es que existen ciertos estados respecto de los cuales, para cualquier persona que estuviera en ellos sería algo malo. Ser ciego es algo malo para cualquiera que esté en tal estado. Del mismo modo, si alguien tiene un disparo en el brazo, esa persona está en un estado malo. Cualquiera que haya causado en otro alguna de esas circunstancias, lo habrá dañado. La tesis no comparativa de dañar, o simplemente TNC, podría sintetizarse como sigue:

TNC: Si el acto de A causa que B esté en un estado malo, entonces A daña a B

La ventaja de esta clase de aproximaciones por sobre las comparativas es que alcanza con afirmar que alguien ha colocado a otra persona en un estado malo para decir que la ha dañado. Conforme a las TNC parece posible explicar por qué el acto de Moni daña a Paola y es moralmente objetable. Esto es así porque como ser sordo es estar en un estado malo y Paola es sorda, dado que Moni causó tal estado, entonces ella la ha dañado. Sin embargo, existen ciertos inconvenientes vinculados a esta aproximación para explicar por qué Moni ha dañado a Paola. El problema surge en los casos donde el agente causa que alguien esté en un mal estado, pero no tan malo como el estado en el que podría haber estado si el acto no se hubiese realizado:

una respuesta afirmativa o negativa, sino que la identidad puede ser indeterminada (Parfit, 1984, 354). Sin embargo, como en el espacio de dos meses de concepción, no sólo el espermatozoide sino también el óvulo hubiese sido diferente, es cierto que la persona que -de hecho- fue concebida en otro momento temporal, nunca habría existido. Derek Parfit denomina a este argumento Tesis de la Dependencia Temporal II: si una persona particular no hubiese sido concebida en el espacio de un mes alrededor del momento en que de hecho fue concebida, de hecho, nunca habría existido $(1984,355)$. Para quién no esté convencido con tal argumento, véase con mayor detalle Parfit,(1984, 351-355) y Boonin (2014, 29-51) 
(El cirujano) Has tenido un grave accidente. Tan grave que si un cirujano no realiza un procedimiento extremo, morirás. Por tanto, el cirujano amputa tu brazo porque es la única manera de salvar tu vida. Lo que el cirujano hace es mejor que cualquier posible alternativa. ¿Te ha dañado el cirujano al amputar tu brazo? ¿Debe responder por ello?

Las dos preguntas son diferentes, y claramente la respuesta a la segunda es negativa. Es menos claro si el cirujano te ha dañado o no con su accionar. Sin embargo, cualquiera de las dos respuestas que se den a esta pregunta son problemáticas para las tesis no comparativas. Si se responde que el cirujano no ha dañado al accidentado, entonces debe explicarse por qué Moni ha dañado a Paola. En los dos casos, lo que hace el agente deja a la víctima mejor que cualquier alternativa posible. Si por el contrario, se responde que en ambos casos se ha dañado a la víctima, pero que en el caso de Moni se es responsable por el daño, en tanto que no se lo es por el caso del Cirujano, entonces debe buscarse una respuesta que explique esa diferencia. Sea cual sea la respuesta, cualquiera que defienda una aproximación no-comparativa debe intentar responder a la siguiente pregunta ¿Por qué el acto de Moni es moralmente incorrecto, en tanto que no lo es el acto del cirujano?

\subsection{La alternativa posible}

Un intento por dar respuesta es el elaborado por Elizabeth Harman (2004 y 2009). Ella afirma que en casos como el de Moni y Paola, parece claro que (a) es incorrecto concebir a Paola porque dado que ella será sorda en el futuro, habrá sufrido un daño. También, que (b) hay razones en contra de concebir a Paola en virtud del daño que reporta para Paola. Sin embargo, (c) dado que en las explicaciones tradicionales, una persona daña a otra sólo si hace que esté en una peor condición de la que podría haber estado si la acción no se realizara, parece que Moni no daña a Paola. Si esta última afirmación (c) es verdadera, entonces la segunda (b) es falsa. Harman, cree que esto es un error. Cree que la última afirmación (c) es falsa y la segunda (b) es verdadera. Su objetivo es encontrar una explicación de por qué la acción de Moni daña a Paola y por qué ello es moralmente incorrecto.

La idea de Harman es sencilla. Se basa en dos afirmaciones: La primera se refiere a lo que significa dañar: (1) "una acción daña a alguien si la acción causa dolor, muerte prematura, daño corporal, disconformidad incluso si ella no hubiese existido si la acción no se hubiese realizado" (Harman, 2004, 92). Como explicación no comparativa, dañar es causar que alguien esté en un estado malo. Segundo (2) "Las razones en contra de dañar son moralmente tan serias que la mera presencia de mayores beneficios para aquellos que son dañados no es en sí mismo suficiente para hacer a los daños permisibles: cuando hay una alternativa en la cual beneficios paralelos pueden ser conferidos sin los daños paralelos, la acción de dañar es incorrecta" (Harman, 2004, 92). Esta segunda afirmación implica que una acción que daña a alguien, por lo tanto es una fuerte razón moral en contra de realizarla. Asimismo que el mero hecho de que el acto de dañar también beneficie a la persona dañada, y la beneficie más de

4 He modificado el ejemplo de Parfit, (1986, 855). 
lo que la daña, es insuficiente para justificar el daño. En suma, la respuesta de Harman puede ser sintetizada como sigue. Se llamará a su propuesta tesis de la alternativa posible 5 , o TAP:

TAP: El hecho de que un acto dañoso cause una cantidad de beneficio mayor a la cantidad de daño que causa a la persona dañada no es suficiente para hacer al acto moralmente permisible si hay una alternativa posible en la que un acto no dañoso podría conferir beneficios comparables a alguien más.

La TAP en el ejemplo del cirujano implica el siguiente razonamiento. Amputarle la pierna al paciente, lo daña. Por tanto, existe una razón en contra de realizar tal procedimiento. Pero al considerar qué razones existen en contra de no realizar el procedimiento, uno observa que las consecuencias serían aún peores. El paciente moriría. Cada razón debe ser sopesada con la otra. Las razones en contra de no realizar la cirugía son mayores que las razones en contra de realizar la cirugía. Sin embargo, la TAP afirma que si una acción produce más beneficios que daños en alguien, esto no es suficiente para hacer a tal acción permisible. Sólo lo será si no existe una alternativa posible donde alguien reciba los beneficios sin los respectivos daños. Efectivamente, en el caso del cirujano esto es lo que ocurre, no hay alternativa posible en la que el cirujano salve la vida de alguien sin los correspondientes daños. Por lo tanto, el acto del cirujano no es moralmente incorrecto.

En el caso de Moni, las cosas son diferentes. Si la sordera es un mal estado para estar en él, esto significa que la acción de Moni de concebir a Paola, la daña. Esto es una razón en contra de concebirla. Sin embargo, concebirla también la beneficia, ya que tendrá una vida que merece la pena ser vivida. Esto es una razón a favor de concebirla. Por tanto, dado que la sordera no es algo tan malo como para hacer de la vida de alguien una que no merece la pena ser vivida, el acto de Moni de concebir a Paola le concede más beneficios que daños. Esto haría suponer que tal acto es permisible. Sin embargo, según la TAP esto no es así. Existe una alternativa posible en la que alguien es beneficiado sin recibir los daños correspondientes. Si Moni espera dos meses y concibe un hijo, el que para propósitos ilustrativos se llamará Coki, ella le concede a alguien más los mismos beneficios que le concedería a Paola, sin los correspondientes daños. Por tanto, la acción de Moni de concebir a Paola es moralmente objetable.

La explicación de Harman parece poder explicar por qué el acto del cirujano no es reprochable y el de Moni de concebir a Paola sí lo es. Por tanto, luce como una buena solución al problema de la no-identidad. Para darle fuerza a su argumento trae el conocido ejemplo del Prisionero Nazi: ${ }^{6}$

(El prisionero Nazi) Un hombre es aprisionado en un campo de concentración Nazi, donde sufre muchos daños. Pero su experiencia en el campo de concentración ha enriquecido su carácter y ha hecho que sea más profundo su entendimiento y valor por la vida, de modo que su vida es, en el balance, mejor que si no hubiese sido aprisionado. El no desea que los Nazis no lo hubieran hecho prisionero, porque él valora lo que ha ganado con la experiencia (Harman, 2004, 99).

5 He tomado esta reconstrucción de Boonin $(2014,85)$

6 El ejemplo es tomado de Woodward $(1986,809)$ quien a su vez lo toma de Adams $(1979,54)$. 
Este ejemplo, parece demostrar que la acción de los Nazis produce un daño impermisible aunque la víctima esté en una mejor condición de la que de otro modo podría haber estado y por más que ella no desee que la acción de los Nazis no se hubiese realizado (Harman, 2004, 99). No estoy seguro que este ejemplo sea una buena defensa de la tesis de Harman. En primer lugar, no veo por qué las tesis comparativas no podrían hacer la misma afirmación. Según ciertas formulaciones de la tesis comparativa contra-fáctica: Si el acto de $\mathrm{A}$ hace que $\mathrm{B}$ esté en algún aspecto en una peor condición de la que podría haber estado si A no hubiese actuado como lo hizo, entonces el acto de A daña a B. En el caso del prisionero Nazi, del mismo modo, él está en un aspecto peor de lo que podría haber estado de otro modo -si los Nazis no hubieran actuado como lo hicieron-, por más que en el balance esté mejor.

En segundo lugar, uno podría afirmar que los Nazis no sabían que el prisionero iba a estar objetivamente mejor por haber sido aprisionado. Que todo lo que importa es que, teniendo en cuenta el conocimiento de los captores, el prisionero iba a estar previsiblemente peor de lo que estaría de otro modo. Harman, retomando un argumento de Woodward $(1987,803)$ afirma que aún en el improbable caso de que los Nazis hubieren hecho lo que hicieron para beneficio del prisionero, uno seguiría pensando que lo que hicieron es reprochable (Harman, 2004, 101). Esto puede ser cierto. Sin embargo, que lo sea no muestra cómo el ejemplo ayuda a apoyar la tesis de Harman cuando se intenta resolver el problema de la No-identidad. El inconveniente en realizar tal paso es el siguiente. Decir que los Nazis han dañado al prisionero y que ello es impermisible parece ser lo mismo que decir que ellos han ocasionado algo malo en el prisionero y que se le ha violado un derecho. Si el prisionero tenía un derecho a no ser dañado, entonces parece posible explicar por qué la acción de los Nazis es impermisible. Del mismo modo, cuando Moni concibe a Paola, su acción al dañarla, parece violar su derecho a tener el sentido de la vista. Por tanto, ambos casos parecen simétricos.

Sin embargo, existe un problema con esta reconstrucción, que ha sido puntualizada por McMahan. La función de los derechos es proteger los intereses de las personas. Esto es crear una barrera que las deje, al menos en un aspecto, mejor que determinado estándar. Esto ocurre en el caso del prisionero Nazi. Pero en el caso de Paola pareciera que los derechos no pueden cumplir esa función (McMahan, 1981:125). Su existencia no hace que la persona que queríamos proteger esté mejor, sino sencillamente, que no exista.

Quizás por estas observaciones, Harman en su escrito del año 2009 realiza una distinción entre lo que ella llama (4) y lo que llama (4*). En el trabajo de ese año, descompone sus dos afirmaciones principales (de 2004) en $\operatorname{tres}^{7}$ y agrega una cuarta:

(4) "Si una acción daña a alguien que no tiene existencia independiente a la acción, entonces el hecho que la acción también beneficie a la persona dañada, y la beneficie más de lo que la daña, no es apto para justificar el daño si no realizar la acción sería igualmente beneficioso para alguien" (Harman, 2009, 139).

7 “(1) Uno daña a alguien si causa en él, dolor, malestar físico o mental, enfermedad, deformidad, discapacidad o la muerte" (2) "Una acción que daña a alguien, por lo tanto es una fuerte razón moral en contra de ella" y (3) "El mero hecho de que una acción dañosa también beneficie a la persona dañada y la beneficie más de lo que la daña, es insuficiente para justificar el daño" (Harman, 2009, 139). 
(4) no es compatible con TAP dado que realiza una distinción donde TAP no lo hace. (4) distingue entre personas que tienen existencia independiente a la acción de dañar y personas que no la tienen. La afirmación que se corresponde con TAP sería:

(4*)"Si una acción daña a alguien, entonces el hecho que la acción también beneficie a la persona dañada, y la beneficie más de lo que la daña, no es apto para justificar el daño si no realizar la acción sería igualmente beneficioso para alguien” $(2009,47)$.

Harman se ve obligada a proponer (4) porque la afirmación (4*) implicada en su tesis, como ella misma reconoce, tiene consecuencias contra-intuitivas. Para justificar el recorte trae un ejemplo donde una persona puede distribuir un beneficio, un helado, por ejemplo. Puede sólo dárselo a una persona. Ben y Sally lo quieren, pero si Sally lo recibe sufrirá una leve migraña. Por lo tanto, si $4^{*}$ es verdadera, afirma Harman, es impermisible que el agente le dé el helado a Sally ${ }^{8}$. En otros casos, 4* trae aparejada consecuencias aún peores. Imagine que un doctor intenta salvar la vida de un paciente, sin embargo le dejará cierto nivel de dolor post-operatorio. Repentinamente aparece otro paciente con una anomalía similar, al cual puede salvar realizando una operación sin dejarle ningún nivel de dolor-post operatorio. ${ }^{9}$ La tesis de Harman (TAP) y $4^{*}$ implican que es impermisible para el doctor salvar al primer paciente. Esto parece implausible. Por tanto, en su trabajo de 2009, Harman afirma que $4 *$ debe ser falsa, lo que es una razón para apoyar a 4. Si esto es así, entonces la tesis de Harman es enteramente ad hoc.

Harman reconoce esta objeción y lanza un argumento para defender el recorte realizado, en favor de la asimetría entre los efectos de los actos sobre las personas actualmente existentes y aquellos que no tienen existencia independiente del acto que las daña. En primer lugar, afirma que en los casos normales ocurre tanto (a) que algo malo le pasa a la persona dañada, y (b) que uno evita la existencia de la persona dañada sin esas cosas malas (Harman, 2009, 146); en tanto que en los casos atravesados por el problema de la no identidad, sólo ocurre lo primero. Por lo tanto es peor dañar a alguien que tiene existencia independiente de la acción que a alguien que no la tenga. Si Moni no concibe a Paola, ella no ha sido impedida de tener una existencia sin esas cosas malas. Del mismo modo, cuando una acción beneficia a alguien que existe, (c) la acción no sólo lo beneficia, sino que (d) si la acción no es realizada evita que la persona obtenga un beneficio. En cambio en los casos atravesados por el problema de la no identidad, solo es cierto lo primero, pero no lo segundo (Harman, 2009, 147). Si Moni no concibe a Paola no hay nadie a quién se haya evitado de obtener un beneficio.

Encuentro difícil ver cómo alguien que adhiere a una tesis no comparativa de dañar a otro pueda hacer estas afirmaciones. (b) y (d) parecen afirmaciones esencialmente comparativas. Aún si se acepta que dañar es ocasionar cosas malas en otro, parece que la gravedad del

8 En particular, acuerdo con Harman en que es permisible darle el helado a Sally, pero no por la distinción que ella marca, sino porque Sally ha consentido en ser dañada. Si así no fuera, entonces no creo que la acción de entregar el helado fuere permisible. Del mismo modo, si Paola consintiera en ser dañada, la acción de Moni sería permisible, no lo sería, en cambio, si Paola no acuerda. Obviamente, en los casos intergeneracionales existe el problema referido a cómo saber si las generaciones futuras consienten o no los daños que sufren. Este tema requiere un análisis diferente.

9 El ejemplo es de Boonin $(2014,91)$ 
daño depende de si esa persona podría haber estado mejor de otra manera. Si eso es posible, el daño es más grave; si no lo es, es más leve. ${ }^{10}$ ¿Cómo puede alguien que adhiere a tesis no comparativas del daño, decir coherentemente que su gravedad depende de asunciones comparativas?

\subsection{Daño como distancia entre dos estados}

Seana Shiffrin intenta explicar por qué está justificado el daño que realiza el cirujano al amputarte la pierna para salvar tu vida, y no lo está el que tiene lugar cuando Moni concibe a Paola. Para ello, comienza afirmando que su posición, a diferencia de las tesis comparativas, "identifica a los daños con ciertas condiciones no comparativas y absolutas" (Shiffrin, 1999, 123). Su explicación de tal idea es la siguiente: “daño implica condiciones que generan una brecha o conflicto importante entre la voluntad y la experiencia de uno, la propia vida entendida en términos más generales, o las circunstancias de cada uno" (Shiffrin, 1999, 123). La propuesta de Shiffrin puede ser llamada como tesis de la brecha entre dos estados (el querido y el real), o simplemente TB2E y sintetizada:

TB2E: Si el acto de A hace que B esté en un estado en el cual B no querría racionalmente estar, entonces A daña a B.

Desde tal conceptualización, entonces, la sordera de Paola es un daño. Del mismo modo lo es para el paciente tener el brazo amputado. En ambos casos puede decirse que un agente racional querría tener una vida sin tales condiciones malas. El argumento de Shiffrin se puede reconstruir del siguiente modo. Existen dos asimetrías de importancia. La primera surge de la diferencia entre la fuerza dada por las razones ejercidas por los daños y la fuerza de las razones derivadas de los beneficios. Generalmente si todo lo demás es igual, dañar, los eventos dañosos y las oportunidades de dañar son más importantes moralmente que los beneficios, los eventos beneficiosos y las oportunidades para beneficiar. Los daños tienen una gran capacidad de generar razones para la acción y tienden a generar razones más fuertes que los beneficios (Shiffrin, 2012, 361). De esta manera, si una acción dañosa también produce un beneficio, esto no es suficiente para justificar tal daño. Hasta aquí, sus afirmaciones son similares a las de Harman. Sin embargo es diferente la razón a la que apela para justificarla. Afirma la autora que la imposición de daños requiere grandes justificaciones, tales como que el daño fue merecido por la víctima, que la imposición era necesaria para evitar un daño mayor, o para vindicar otro daño, o, al menos requiere el consentimiento del dañado. En cambio, tales razones no son necesarias para otorgar beneficios (Shiffrin, 2012, 362).

La segunda asimetría surge del contraste entre lo qué es permisible hacer para evitar o aliviar daños y lo que es permisible hacer para otorgar o proteger beneficios (Shiffrin, 2012, 363). Shiffrin, distingue entro lo que llama "puro beneficio" (1999, 124 y 2012, 364) y aquello que se ha denominado como "beneficio preventivo" (Hanser, 2009, 183). Existe un beneficio preventivo cuando la acción de un sujeto evita que otro sufra alguna clase de daño. En estos casos parece que es permisible infligir un pequeño daño necesario para

10 Boonin proporciona otras tres razones para rechazar tal argumento. Véase Boonin (2014, 351-356). 
evitar uno significativamente mayor. El daño evitado sería el llamado beneficio preventivo. Un puro beneficio, en cambio, sólo implica agregar algo bueno a la condición de alguien. Estos últimos, no evitan, suprimen o alivian ninguna clase de daño. Por tanto es menos claro que sea permisible infligir la misma clase de pequeño daño para otorgar un puro beneficio significativamente mayor. (Shiffrin, 2012, 364). Imagine que alguien empuja a un peatón de manera que golpea su cabeza contra una pared, porque de lo contrario una gran maceta que estaba cayendo de un balcón podría haberlo aplastado. Parece evidente que el daño que se le produce es permisible debido a que se ha evitado que al peatón le suceda algo malo (algún daño aún más grave). En cambio, pareciera que empujar a un peatón de manera que golpee su cabeza contra una pared no es permisible si alguien había fehacientemente prometido que si se realiza tal acto, se recompensará al peatón con un millón de pesos. Los beneficios preventivos (primer caso) pueden justificar la imposición de daños, aunque los puros beneficios no (segundo caso).

En los casos atravesados por el problema de la no identidad, la tesis de Shiffrin implica lo siguiente. El acto de Moni de concebir a Paola la daña porque la coloca en una mala condición. Esto es así, porque ella será sorda al nacer. Pero el acto de Moni de concebir a Paola también le concede un beneficio dado que le permite tener una vida que vale la pena ser vivida y muchas cosas buenas le pasarán. Puede suponerse que los beneficios que Moni le concede a Paola serán mayores que los daños. Sin embargo, para que estos beneficios sean capaces de justificar el daño impuesto, deben ser beneficios preventivos. Será de esta clase, si concebir a Paola la protege a ella de algo malo que le ocurriría si ella no hubiese sido concebida. Este no parece ser el caso. El beneficio que recibe Paola luce más como un puro beneficio. La vida no es algo que haya sido otorgado que prevenga que Paola esté en un mal estado. De esta manera, la tesis de Shiffrin explicaría por qué el acto de Moni daña a Paola y por qué es impermisible.

Para que la tesis sea plausible y, por tanto, pueda resolver el problema de la no-identidad, debería implicar que el daño que produce el cirujano que amputa la pierna de su paciente para salvar su vida está justificado. Desde que tener una sola pierna es estar en un estado malo, el cirujano ha dañado a su paciente cuando lo coloca en tal situación. La operación, asimismo, le produce al paciente un beneficio mayor que el daño que le ocasiona. El paciente puede seguir viviendo. El beneficio que el cirujano le produce al paciente es evitar la muerte que, de otra manera, habría sufrido. En este caso, lo ocurrido luce más como un beneficio preventivo que como un puro beneficio. Por tanto, el acto del cirujano evita que algo malo le ocurra al paciente, de modo que el daño producido está justificado. Siendo esto así, la tesis de Shiffrin implica que el acto de Moni de dañar a Paola es moralmente incorrecto, y debe responder por ello, en tanto que no lo es el acto del cirujano que amputa la pierna para salvarle la vida al paciente. Parece que el problema de la no-identidad ha sido resuelto. ¿Es esta una buena solución?

Para justificar su tesis, la autora trae dos casos denominados "el rescate y los lingotes de oro", los que serían análogos al caso del cirujano y al de Moni y Paola -respectivamente:

(El rescate) Victoria se está ahogando. Si el guarda vidas no la rescata, ella sufrirá un gran daño cerebral (o la muerte) por pérdida de oxígeno. Para rescatarla, el guarda-vidas debe quebrar el brazo de Victoria, dado que está atorado con una roca. 
El guarda-vidas rescata a Victoria. Como resultado, ella ha sido salvada del daño cerebral (o la muerte) y tiene un brazo dañado. ${ }^{11}$

(Los lingotes de oro) Imagine a un personaje adinerado -Rodrigo- quien vive en una isla. Él está ansioso por un proyecto. Decide otorgar algo de su dinero a sus vecinos de la isla adyacente. Desafortunadamente, por circunstancias históricas, no se les permiten a los miembros de la isla del personaje adinerado visitar a la otra. Por lo que, para implementar su proyecto, decide rentar un avión y arrojar lingotes de oro desde él, cada uno de los cuales tiene un valor de 10 millones de pesos. Una persona, lamentablemente, es golpeada por un lingote. El impacto quiebra su brazo ${ }^{12}$.

Los casos intentan mostrar lo siguiente. Que el acto del guarda-vidas daña a Victoria. Sin embargo el daño producido está justificado, y por tanto, no debe responder por él. En cambio, en el caso de los lingotes de oro el personaje adinerado daña al desafortunado, y debe responderse por ello. La razón está dada porque en este último caso la acción que provoca el daño produce un "puro beneficio". En tanto que en el caso del rescate, la acción que provoca un daño también ocasiona un "beneficio preventivo" De esta forma, aunque en los dos casos se producen daños en otras personas, sólo debe responderse por el ocasionado por los lingotes de oro. En este supuesto la acción realizada no evita que el habitante desafortunado de la isla, sufra ninguna clase de daño. Por lo tanto, según el análisis de Shiffrin, el habitante adinerado -Rodrigo- debería compensar al desafortunado por el daño sufrido. Lo que, obviamente, no es necesario en el caso del rescate.

Coincido con una de las razones que D. Boonin esgrime para afirmar que los casos no son una buena defensa de la tesis de Shiffrin ${ }^{13}$. La diferencia entre beneficios preventivos y puros no es la única explicación de la diferencia entre el caso del rescate y el de los lingotes de oro. Los daños y beneficios en el caso del rescate son aproximadamente equiparables, ambos tienen que ver con el bienestar físico. Pero en el caso de los lingotes de oro, los daños y beneficios son menos fácil de comparar: se daña a la integridad física y se beneficia el patrimonio. Es posible que la diferencia en conmensurabilidad haga al acto del rescatista moralmente permisible y el del personaje adinerado impermisible (Boonin, 2014, 96). Para probar su punto, Boonin modifica uno de los ejemplos para hacer a los daños y beneficios comparables:

11 He modificado ligeramente el ejemplo de Boonin $(2014,95)$

12 He modificado levemente el ejemplo de Shiffrin $(1999,127)$

13 La otra razón que Boonin esgrime no me parece correcta. Él afirma que no es tan claro que el habitante adinerado le deba una compensación al desafortunado que le quiebra el brazo, dado que podría obtenerla del lingote que ahora posee. Asimismo, que del hecho de que le deba una compensación no se sigue que el acto del personaje adinerado sea impermisible. Es permisible romper el vidrio de una casa para entrar y salvarse de una tormenta, aunque luego se le deba una compensación al dueño. Del mismo modo, argumenta, puede ser permisible dañar al desafortunado y luego compensar por el daño (Boonin, 2014, 95-96). Creo que este argumento confunde el motivo por el cual el acto es permisible en los dos casos. Si el motivo es diferente, la analogía no sirve y, por tanto, tampoco el argumento. Romper el vidrio de una casa y entrar es permisible porque en caso de no hacerlo el agente se vería dañado gravemente por la tormenta. En cambio, nada por el estilo sucede en el caso de los lingotes de oro. Si el personaje adinerado no arroja los lingotes de modo que daña al desafortunado, nada le ocurre al primero, de modo que la justificación en ambos casos no puede ser la misma. 
(Dos lingotes) Un personaje acomodado (Walter) desea hacerle un regalo a Saul. El único regalo que puede darle es un lingote de oro de \$ 10 millones. La única manera de dárselo a Saul es dejarlo caer sobre una de sus posesiones: un lingote de oro de $\$$ 500. Si Walter lo hace, el lingote de oro más pequeño será destruido. Walter no puede comunicarse con Saúl a tiempo, de modo que no puede pedir su consentimiento. Walter deja caer el gran lingote de oro sobre el pequeño lingote de Saúl, poniéndolo a este último en posesión del gran lingote. Como resultado Saúl ahora tiene un lingote de $\$ 10$ millones y no uno de $\$ 500$.

Como en el primer caso, Walter le concede un puro beneficio a Saúl. Pero en éste a diferencia del otro, los daños y beneficios son fácilmente conmensurables. Mientras es plausible pensar que en el primer caso el personaje adinerado ha hecho algo incorrecto al quebrar el brazo del desafortunado, en este caso es más difícil de sostener que el daño que Walter le produce a Saul es incorrecto. Del mismo modo, si alguien deposita 10 millones de pesos en mi cuenta bancaria y debo pagar 500 pesos de impuestos por ello, sería raro sostener que he sido dañado (Boonin, 2014, 97). El argumento de Boonin muestra que el acto del personaje adinerado del primer caso es incorrecto por producir un daño incomparable con el beneficio que otorga, y no por producir un beneficio preventivo capaz de justificar el daño. Si esto es así, los ejemplos aportados por Shiffrin no pueden justificar la distinción entre la impermisibilidad de un daño cuando se otorga a su vez un beneficio puro y la permisibilidad de cuando se otorga a su vez un beneficio preventivo. Por tanto su tesis no parece tan sólida como antes para resolver el problema de la no identidad.

Pero esto no es todo. El caso de los dos lingotes parece defender de manera convincente que a la hora de justificar o no la imposición de ciertos daños es relevante si estos pueden ser aproximadamente comparables con los beneficios que otorga. El caso de Paola, parece ser más similar al caso de los dos lingotes de oro que al de uno solo. Si bien la vida para Paola es un puro beneficio, es uno que puede ser comparado aproximadamente con el daño que le representa la sordera. Ambos tienen que ver con su bienestar físico y psíquico. Y, al igual que en el caso de los dos lingotes de oro, en casos de este tipo parece que los grandes beneficios pueden sopesar al daño. De modo que, conforme a este argumento, el problema de la no-identidad no ha sido resuelto. El acto de Moni de concebir a Paola sigue siendo moralmente no objetable.

\section{Dañar a otro: una aproximación superadora}

Existe otra posibilidad, dentro del no-comparativismo en orden a resolver el problema. El mejor candidato para cumplir este rol son las llamadas tesis del umbral. De acuerdo a ellas "una acción daña a una persona si a consecuencia de tal acción la persona cae por debajo de un umbral normativamente definido" (Meyer \& Roser, 2009, 228). La idea general detrás de la interpretación del umbral del daño es que se tiene el deber general de no causar que las personas estén en una peor condición que aquella en la que deberían estar (Meyer \& Roser, 2009, 229). La tesis del umbral o $T U$, puede ser resumida como sigue:

TU: Si el acto de A hace que B caiga debajo de un umbral específico, entonces A daña a B. 
Según esta posición, si, por ejemplo, el umbral está marcado por tener un sentido de la audición normal, TU implica que, dado que Moni al concebir a Paola hizo que ella sea sorda, desde que ser sorda cae debajo del umbral, Moni ha dañado a Paola. Como se observa tal posición se encuentra en el mismo marco teórico que las anteriores. Por lo tanto, para ser superadora debería encontrar una razón diferente a la aportada por aquellas a la hora de distinguir la objetabilidad de la acción realizada por Moni y la in-objetabilidad de la acción realizada por el cirujano. Según TU, si causar un daño es colocar a alguien por debajo de cierto umbral, entonces el cirujano que, en nuestro ejemplo, mejora la condición de un paciente pero lo deja aún por debajo de él, lo ha dañado. Esto parece implausible. Se ha visto que ni la respuesta de Harman ni la de Shiffrin pueden solucionar este inconveniente. Sin embargo existe otra posibilidad, que, de ser satisfactoria podría hacer a las tesis no-comparativas plausibles en orden a resolver el problema de la no-identidad. Según los partidarios de TU, en la situación descrita, el cirujano no puede evitar dañarte. Haga lo que haga dejará a su paciente por debajo del umbral. Como esto es así, afirmar que existe una obligación de no causar daño no tiene sentido. A nadie se le puede exigir que haga aquello que está fuera de sus posibilidades. Sin embargo, sí se les puede exigir que hagan aquello que menor cantidad de daño produzca (Meyer, 2003, 153). Es decir que uno evite que alguien esté en una peor condición bajo el estándar de aquella que se causó. Si no lo hace, habrá dañado más al sujeto pasivo. Esta respuesta parece satisfactoria, sin embargo, se puede objetar que tal afirmación no está implicada en la tesis del umbral, sino en aproximaciones comparativas, razón por la cual debería darse una razón extra para sostenerla.

La segunda objeción a la TU es que es infra-inclusiva. Si alguien está peor de lo que estaría de otro modo, pero todavía por arriba del umbral, parece que de todos modos ha sido dañado ${ }^{14}$. Aquí, otra vez, una aproximación comparativa da la respuesta correcta en detrimento de posiciones como la TU. Una defensa de la TU, nuevamente, puede apelar a la obligación de dejar a la persona a quienes las consecuencias afectan lo más lejos posible del umbral. Estas consideraciones hacen que parezca plausible adoptar una visión combinada ${ }^{15}$, o TComb (Meyer, 2003, 154-155). De acuerdo con ella:

TComb: Si A realiza un acto que o

(1) causa que B caiga por debajo de cierto umbral y, si no es posible evitar causar daño en ese sentido, A no minimiza el daño en B; o

(2) causa que B esté peor de lo que estaría si A no hubiese actuado como lo hizo

entonces A daña a B.

14 Imagínese que: Tim ha golpeado a Derek, ganador del premio Rolf Schock en filosofía y lógica, en la cabeza. Como resultado Derek tiene ahora una inteligencia promedio y sus trabajos académicos han bajado su nivel. ¿Ha dañado Tim a Derek? La respuesta parece ser afirmativa, lo que es un problema para las tesis del umbral, desde que tener una inteligencia promedio siempre debe quedar por encima de él.

15 Jeff McMahan, por ejemplo, también considera que es inevitable apoyar una aproximación pluralista o combinada del daña que involucre tanto aspectos comparativos como no comparativos (McMahan, 2012, 4) Sin embargo, más allá de esta afirmación y la ejemplificación de diferentes casos donde alternativamente uno u otra posibilidad funcionan, no da demasiados argumentos. 
Pareciera que esta tesis podría resolver toda clase de casos que se puedan presentar. De esta manera, sería posible explicar al mismo tiempo la objeción al obrar de Moni sobre la base de una tesis no-comparativa del daño y la in-objetabilidad al obrar del cirujano sobre la base de una tesis comparativa del daño. Sin embargo, más allá de esa consecuencia plausible, no se han dado demasiados argumentos para afirmar que adoptar tal conceptualización del daño está justificado. Asimismo, quedan muchos interrogantes abiertos: ¿Por qué en determinados casos se debe aplicar una aproximación comparativa y en otros una no-comparativa? $\mathrm{O}$ ¿es alguna de las dos aproximaciones prioritaria? o ¿Qué clase de daños son peores, los comparativos o los no comparativos?

Adicionalmente, el problema con esta aproximación es que, en primer lugar, dado que se intenta encontrar una tesis del daño que pueda cumplir funciones prácticas, debería poder explicitarse cómo se conforma el umbral, es decir qué es aquellos que las personas deberían tener y, en ausencia de lo cual, se puede sostener que han sido dañadas (Smajdor, 2014, 14). Asimismo, si para identificar al daño implicado en el principio del daño se debe conocer lo que las personas tienen derecho a tener, entonces la discusión relevante ya no sería en términos del principio del daño, sino en términos de una teoría de la justicia. Algunos autores han criticado a los defensores de respuestas basadas en el daño por implicar tal conclusión (Holtung, 2002, 385). Prueba de ello es que el mismo Meyer, al momento de tener que especificar el umbral, discute la formulación que de él puede hacerse desde concepciones suficientaristas, igualitaristas o prioritaristas (Meyer, 2014, 13-17). ${ }^{16}$

\section{Conclusión}

En este trabajo se intentó analizar si las tesis no-comparativas del daño pueden resolver el problema de la no-identidad. El resultado ha sido que ninguna de estas posiciones, por sí sola, puede dar una respuesta satisfactoria al inconveniente y explicar por ejemplo por qué, en casos como el analizado, Moni ha dañado a Paola. De manera que la respuesta al interrogante inicial es negativo. Las tesis no-comparativas del daño no pueden resolver el problema de la no-identidad.

Sin embargo, también se ha visto que el problema podría ser resuelto si fuera posible construir una tesis combinada, donde el comparativismo corrija los defectos de no-comparativismo. Este trabajo ha mostrado un esquema sobre la forma que debería tener tal tesis. Sin embargo, no se ha podido identificar un principio que la pueda justificar. No obstante, ello no significa que el trabajo sea condenado al fracaso. Ahora se conoce lo que este principio debe implicar, de manera tal que tal vez sea más fácil identificarlo. ${ }^{17}$

16 Tal vez, como me ha sugerido un árbitro anónimo, la inmoralidad del acto de Moni deba buscarse en tesis diferentes a las aportadas por aquellas basadas en el daño a Paola. Pero un análisis de tal posibilidad excede los límites de este trabajo.

17 Agradezco a los profesores Hugo Seleme, Miguel A. Rodilla, Guillermo Lariguet, José Peralta y Fabián Balcarce por sus valiosos comentarios. Agradezco también a Romina Frontalini y Ramiro Moyano con quienes he discutido reiteradas veces el argumento de este trabajo. Asimismo a L. Misseri, A. Vercellone, E. Olivares, C. Fatauros, y demás miembros del programa de Ética y Teoría Política (CIJyS-UNC) quienes en diversas ocasiones me han presentado varias objeciones. 


\section{Bibliografía}

Adams R. M. (1979) "Existence, Self Interest, and the problem of evil”, Noûs, Vol. $3 \mathrm{~N}^{\circ}$ 1, pp. 53-65.

Boonin, David (2014) The Non-Identity Problem and the Ethic of Future People, Oxford University Press.

Feinberg, Joel (1994) "Wrongful Life and the Counterfactual Element in Harming" en: él mismo, Freedom and Fulfillment: philosophical essays, New Jersey, Princeton University Press, pp. 3-36.

Hanser, Matthew (2009) "Harming and procreating" en Roberts, M \& Wasserman D. (Ed.) (2009) Harming Future Peoples: Ethics, Genetics and the Non Identity Problem, New York, Springer, pp. 179-199.

Harman, Elizabeth (2004) "Can we harm and benefit in creating?", Philosophical Perspectives, 18, Ethics, 2004, pp. 89-113.

Harman, Elizabeth (2009) "Harming as Causing Harm" en Roberts, M \& Wasserman, D. (Ed.) (2009) Harming Future Peoples: Ethics, Genetics and the Non Identity Problem, New York, Springer, pp. 137-151.

Holtung, Nils (2002) “The Harm Principle”, Ethical Theory and Moral Practice, Vol. 5. No. 4 (Dec., 2002), pp. 357-389.

McMahan, Jeff (1981) "Problems of Population Theory”, Ethics, 92 N$^{\circ} 1$ (Oct. 1981), 96-127.

McMahan, Jeff (2012) "Causing People to Exist and Saving People’s Lives", Ethics, Springer, 13.set.2012

Meyer, Lukas (2003) "Past and Future: The Case for a Threshold Notion of Harm" in Meyer, L. -Paulson, S \& Pogge T. Rights, Culture, and the Law: Themes from the Legal and Political Philosophy of Joseph Raz, Oxford University Press, pp. 143-159.

Meyer, Lukas \& Roser, Dominic (2009) "Enaugh for the Future" in Gosseries A. \& Meyer, L. (2008) Intergenerational Justice, Oxford University Press, pp. 219-248.

Meyer, Lukas, (2014): "Intergenerational Justice", The Stanford Encyclopedia of Philosophy (Winter 2014 Edition), Edward N. Zalta (ed.), URL = <http://plato.stanford.edu/ archives/win2014/entries/justice-intergenerational/>.

Parfit, Derek (1981), "Energy and the Further Future" en Center for Philosophy and Public Policy, University of Maryland (1981), pp. 112-121.

Parfit, Derek (1982), "Future Generations: Further Problems" en Philosophy \& Public Affair, Vol. 11, N 2 (Spring, 1982), 113-172.

Parfit, Derek (1984), Reasons and Persons, Oxford, Clarendon Press.

Parfit, Derek (1986) “Comments” Ethics, vol. 96, No 4. (Jul., 1986), 832-872.

Raz, Joseph (1986) The Morality of Freedom, Clarendon, Oxford University Press.

Shiffrin, Seana V. (1999) "Wrongful Life, Procreative Responsibility and the Significance of Harm”, Legal Theory, Vol. 5, (1999) Cambridge University Press, pp.117-148

Shiffrin, Seana V. (2012) "Harm and Its Moral Significance", Legal Theory, Vol. 18 Sep. (2012), Cambridge University Press, pp. 357-398.

Smajdor, Anna (2014) "How Useful is the concept of the Harm Threshold in reproductive ethics and law?" in Theor Med Bioeth, Springer, 09, August 2014, pp. 1-16.

Woodward, James (1986) “The Non-Identity Problem”, Ethics, vol.96, No 4. (Jul., 1986), 804-831. Woodward, James (1987) "Reply to Parfit", Ethics, Vol 97, N 4 (Jul, 1987) pp. 800-816. 